\title{
CELIAC DISEASE DIAGNOSED BY VIDEO CAPSULE ENDOSCOPY
}

\section{de Vicente Ortega A, Tercero Lozano M, Jamal Ismail S}

UGC Aparato Digestivo, del Complejo Hospitalario de Jaén. Jaén.

\section{Resumen}

La celiaquía es una enfermedad multiorgánica autoinmune crónica, que afecta al intestino delgado en individuos genéticamente predispuestos, cuya prevalencia se está viendo aumentada.

Exponemos un caso de celiaquía, en el que gracias a la obtención de una imagen típica por videocapsula endoscópica, se consigue una alta sospecha que permite completar el diagnóstico.

Como conclusión, queremos resaltar la importancia de la imagen típica de atrofia vellositaria para la cual la videocápsula endoscópica es muy útil, aunque deba confirmarse histopatológicamente.

Palabras clave: anemia, videocapsuloendoscopia, celiaquía.

\section{CORRESPONDENCIA}

Alicia de Vicente Ortega

Complejo Hospitalario de Jaén

23007 Jaén

aliciavicor@gmail.com

Fecha de envío: 26/07/2020

Fecha de aceptación: 27/09/2020

\section{Abstract}

Celiac disease is a chronic autoinmune multiorganic disorder that affects the small intestine in people who are genetically predisposed. Its prevalence is increasing.

We described a celiac disease case, in which the typical image of duodenum mucosa obtained by video capsule endoscopy, allows us to complete the diagnosis.

As conclusion, we pretend to enhance the utility of the video capsule endoscopy in its diagnosis, which can show the typical image of villous atrophy, although it must be confirmed histopathologically.

Keywords: anemia, video capsule endoscopy, celiac disease.

\section{Introducción}

La enfermedad celíaca se trata de una enteropatía crónica autoinmune, producida por una reacción inmunológica desencadenada por factores ambientales como el gluten, en individuos genéticamente predispuestos $^{1,2}$. 
Es una patología frecuente a nivel mundial cuya prevalencia ha aumentado significativamente en los últimos años, alcanzando en países occidentales el $1 \%$ de la población general ${ }^{1,2}$.

En los adultos la presentación puede ser asintomática, complicando el diagnóstico. Los síntomas clásicos son la diarrea, dolor abdominal y pérdida de peso ${ }^{3}$.

El diagnóstico se puede realizar con criterios como la clínica típica, positividad de anticuerpos antiendomisio y antitransglutaminasa, positividad de genotipos HLA-DQ2 y DQ8, visualización de atrofia vellositaria; pero el diagnóstico definitivo se establece con alteraciones histopatológicas en biopsias ${ }^{3}$.

El tratamiento consiste en eliminar el gluten de la dieta ${ }^{1,2}$, tras lo cual se normaliza la mucosa intestinal y desaparecen los síntomas.

\section{Caso clínico}

Mujer de 38 años, con antecedente personal de diabetes mellitus tipo I, que comienza a estudiarse desde su centro de salud por anemia microcítica ferropénica. Asocia pérdida de peso sin otra clínica acompañante. Fue estudiada desde distintos servicios, como ginecología y hematología, sin evidenciar causa.

La exploración física es anodina, salvo palidez cutánea. En la analítica destacaba: hemoglobina $8,1 \mathrm{~g} / \mathrm{dl}$, hematocrito $29,5 \%$, VCM 74,5fL, hierro $8 \mu \mathrm{g} / \mathrm{dL}$ ferritina $3,1 \mathrm{ng} / \mathrm{mL}$ y ácido fólico 1,5 $\mathrm{ng} / \mathrm{mL}$, anticuerpos $\lg \mathrm{A}$ antiendomisio negativos y anticuerpos $\lg \mathrm{A}$ antitransglutaminasa $13 \mathrm{U} / \mathrm{mL}$.

Se le pautó tratamiento con hierro oral de forma empírica sin presentar mejoría.

Como pruebas complementarias se realizó ecografía, gastroscopia y colonoscopia; todas ellas normales sin visualizar alteraciones ni lesiones. En este primer estudio no se tomaron biopsias por problemas técnicos en relación con mala tolerancia de la paciente a exploraciones invasivas.

Posteriormente, se realiza videocapsuloendoscopia estableciendo lesiones intestinales, visualizadas en duodeno distal, en relación con malabsorción intestinal; celiaquía como primer diagnóstico (Figuras 1 y 2 ).

Tras ello, se realiza nueva analítica a los 6 meses donde destaca el ascenso de anticuerpos IgA antitransglutaminasa $350 \mathrm{U} /$ $\mathrm{mL}$, y se evidencia que es portadora del heterodímero HLA-DQA105/ DQB102 (DQ2.5). Con todo ello, se repite gastroscopia bajo sedación con toma de biopsias de varias zonas de mucosa duodenal. Los resultados anatomopatológicos confirman atrofia vellositaria Marsh III, estableciendo el diagnóstico sospechado de celiaquía.

De este modo, la imagen típica de atrofia vellositaria por videcocapsuloendoscopia nos ayuda a tener una alta sospecha y así consigue iniciar el diagnóstico. Por ende, se puede tratar adecuadamente.

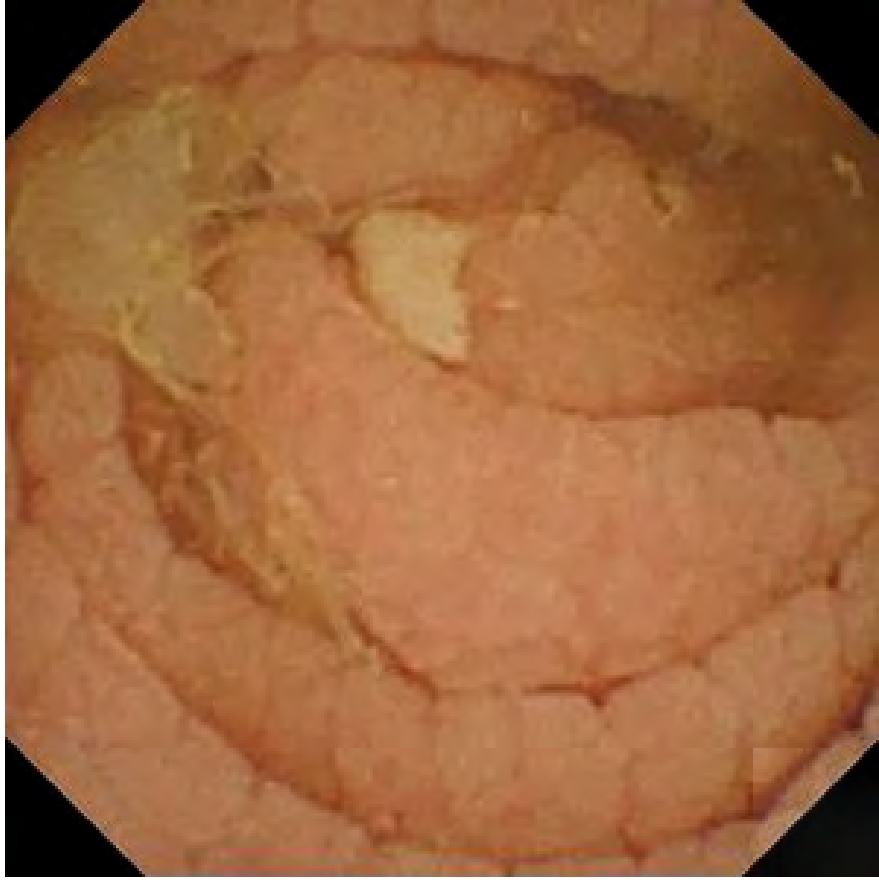

Figura 1

Mucosa de duodeno visualizada por videocapsuloendoscopia. Desde duodeno distal hasta yeyuno distal, mucosa con atrofia de vellosidades y pliegues festoneados, de aspecto mosaico nodular.

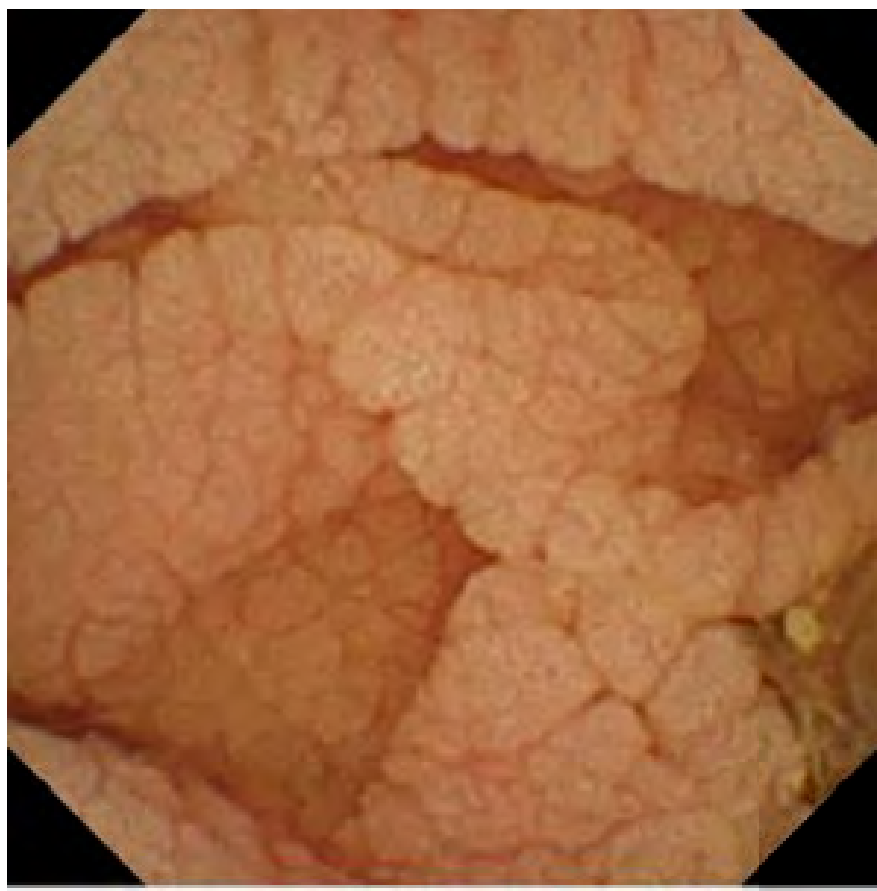

Figura 2

Mucosa de yeyuno visualizada por videocapsuloendoscopia. Desde duodeno distal hasta yeyuno distal, mucosa con atrofia de vellosidades y pliegues festoneados, de aspecto mosaico nodular. 


\section{Discusión}

La celiaquía es una enfermedad multiorgánica autoinmune crónica que afecta al intestino delgado en individuos genéticamente predispuestos, determinada por alelos HLA-DQ2 y DQ8 ${ }^{1}$. Existen algunas enfermedades, como la diabetes mellitus tipo I, enfermedad tiroidea autoinmune, deficiencia selectiva de IgA, etc. que están asociadas con un mayor riesgo de padecerla ${ }^{1,3}$. En el caso que presentamos, el único antecedente de la paciente se trata de diabetes tipo I.

Los hallazgos diagnósticos claves incluyen alteraciones histopatológicas características. Normalmente se evidencian mediante endoscopia con biopsias ${ }^{3}$.

Sin embargo, la videocapsuloendoscopia es un método útil para analizar el trayecto gastrointestinal ${ }^{4,5}$, y detectar alteraciones patológicas como atrofia vellositaria en intestino delgado, una característica de la celiaquía tal y como vemos en nuestro caso.

\section{Bibliografía}

1. World Gastroenterology Organisation. WGO Global Guidelines: Celiac disease; 2016.

2. Domsa E. M, Berindan-Neagoe I, Para I, Munteanu L, Matei D, Andreica V. Celiac disease: a multi-faceted medical condition. J Physiol Pharmacol. 2020; 71(1): 3-14

3. Rej A, Aziz I, Sanders D.S. Coeliac disease and noncoeliac wheat or gluten sensitivity. Journal of Internal Medicine. 2020, doi: 10.1111/joim.13120

4. Ciaccio E. J, Bhagat G, Lewis S. K, Green P. H. Extraction and processing of videocapsule data to detect and measure the presence of villous atrophy in celiac disease patients. Computers in Biology and Medicine; 78. Elsevier Ltd 2016; 97-106.

5. Valverde López F, Jiménez Rosales R, Rodríguez Sicilia M. J, Martín Rodríguez M. M, Sánchez Capilla D, Cabello Tapia M. J, et al. Utilidad de la cápsula endoscópica en estudio del intestino delgado. RAPD 2017; 40 (5): 211-215. 